\title{
Test the mergers of the primordial black holes by high frequency gravitational-wave detector
}

\author{
Xin $\mathbf{L i}^{\mathrm{a}}$, Li-Li Wang ${ }^{\mathrm{b}}, \mathbf{J i n} \mathbf{L i}^{\mathrm{c}}$ \\ Department of Physics, Chongqing University, Chongqing 401331, China
}

Received: 21 June 2017 / Accepted: 11 September 2017 / Published online: 22 September 2017

(C) The Author(s) 2017. This article is an open access publication

\begin{abstract}
The black hole could have a primordial origin if its mass is less than $1 M_{\odot}$. The mergers of these black hole binaries generate stochastic gravitational-wave background (SGWB). We investigate the SGWB in high frequency band $10^{8}-10^{10} \mathrm{~Hz}$. It can be detected by high frequency gravitational-wave detector. Energy density spectrum and amplitude of the SGWB are derived. The upper limit of the energy density spectrum is around $10^{-7}$. Also, the upper limit of the amplitude ranges from $10^{-31.5}$ to $10^{-29.5}$. The fluctuation of spacetime origin from gravitational wave could give a fluctuation of the background electromagnetic field in a high frequency gravitational-wave detector. The signal photon flux generated by the SGWB in the high frequency band $10^{8}-10^{10} \mathrm{~Hz}$ is derived, which ranges from 1 to $10^{2} \mathrm{~s}^{-1}$. The comparison between the signal photon flux generated by relic gravitational waves (RGWs) and the SGWB is also discussed in this paper. It is shown that the signal photon flux generated by the RGW, which is predicted by the canonical single-field slow-roll inflation models, is sufficiently lower than the one generated by the SGWB in the high frequency band $10^{8}-10^{10} \mathrm{~Hz}$. Our results indicate that the SGWB in the high frequency band $10^{8}-10^{10} \mathrm{~Hz}$ is more likely to be detected by the high frequency gravitational-wave detector.
\end{abstract}

\section{Introduction}

The black hole, as one of the predictions of general relativity, has been discussed intensively by physicists. Besides the formation of mini black holes [1] in high energy collisions, the black hole can be formed through gravitational collapse. The gravitational collapse of a heavy star could form a stellar black hole [2]. The gravitational collapse of a primor-

\footnotetext{
a e-mail: lixin1981@cqu.edu.cn

b e-mail: 20152702016@cqu.edu.cn

c e-mail: cqujinli1983@cqu.edu.cn
}

dial overdensity in the early universe could form primordial black holes (PBHs) [3-8]. The PBH has been considered as a possible candidate for dark matter [9-12]. At present, the experimental results for direct searching weakly interacting massive particles, i.e. the main candidate for dark matter, are null $[13,14]$. It motivates physicists to consider other dark matter candidates, such as the PBHs.

The traditional way of searching the black hole depends on indirect observations, such as the accretion disk [15]. Recently, the gravitational wave (GW) has been detected directly by the Advanced LIGO detectors, i.e. GW150914, GW151226 and GW170104 events produced by the mergers of two black holes (BHs) [16-18]. The gravitational wave could be used as a new probe to observe celestial bodies, especially the black hole. It is interesting to distinguish the stellar black hole and the PBH by gravitational waves. The no hair theorem states that the black hole only has three properties, namely, mass, electric charge and spin [19]. Also the mass of the black hole, other than the electric charge and spin of black hole, can be precisely measured by astronomical observations. Thus, the mass of a black hole can be used to distinguish a PBH and a stellar black hole. The upper limit of neutron stars could be 3 solar masses [20]. Therefore, the black hole could have a primordial origin if its mass is less than $1 M_{\odot}$. The $\mathrm{PBH}$ could be formed by several mechanisms [21-32]. The PBH can be formed from the Planck time $10^{-43}$ to $1 \mathrm{~s}$ [33], and the corresponding mass of the black hole ranges from $10^{-5} \mathrm{~g}$ to $10^{5} M_{\odot}$. Due to the Hawking radiation [34], a PBH with mass less than $10^{-18} M_{\odot}$ will evaporate in a time less than the age of our universe. Thus, a black hole could have a primordial origin if its mass ranges from $10^{-18} M_{\odot}$ to $1 M_{\odot}$. Nevertheless, one cannot decide on the origin of these black holes producing GW150914, GW151226 and GW170104 only by their mass. The black holes are of a astrophysical origin [35-37] or primordial origin [38-44] - that is still under debate. 
SGWBs could arise from the incoherent superposition of contributions from many independent and unresolved gravitational-wave sources. It could be generated by corecollapse supernovae [45], rotating neutron stars [46], coalescing stellar binaries [47-51], coalescing black hole binaries [52-59] and magnetars [60]. The SGWB generated by merging black hole binaries could be detected at Advanced LIGO's projected final sensitivity [61]. It is interesting to test the SGWB generated by merging of the binary PBH on different kinds of observatories of gravitational wave, such as Advanced LIGO [61], Pulsar Timing Arrays [6265], eLISA [66] etc. As regards the $\mathrm{PBH}$, as a possible candidate of dark matter, its abundance in dark matter has been constrained from a variety of observations [33]. It is shown in Ref. [33] that the mass of PBH range from $10^{-16} M_{\odot}$ to $1 M_{\odot}$ could have a larger abundance, namely, the upper limit of the constrained abundances is larger than $10^{-2}$. It is worth investigating the signal of gravitational waves generated by mergers of the PBH binary in this mass range. On the other hand, black hole binaries in this mass range cannot have a astrophysical origin. If the corresponding SGWB is detected, it will help us to understand the primordial origin of black holes.

The merging frequency of the binary black hole is inversely proportional to the chirp mass of the binary black hole $[67,68]$. While the chirp mass of the binary black hole equals $1 M_{\odot}$, the merging frequency is close to $10^{4} \mathrm{~Hz}$, which is the upper bound of the detected frequency ranges of Advance LIGO. Thus, one should use a high frequency detector to search the SGWB generated by mergers of the PBH binary where its chirp mass is less than $1 M_{\odot}$. A GW detector has been proposed to detect the GW with high frequency, $10^{8}-10^{14} \mathrm{~Hz}$ [69]. Such a GW detector applies the electromagnetic perturbation effects produced by high frequency GWs to detect GWs. This graviton-to-photon transition was first suggested by Gertsenshtein [70-72]. A different gravitational origin that could generate high frequency GWs has been discussed [73-76]. Besides the SGWB, RGWs [77,78] that were produced in the inflationary stage of the universe are also an important target of high frequency GW detector. Also, both the SGWB and the RGW could come from any direction of the universe. Therefore, to study the SGWB generated by the merger of the PBH binary, it is important to distinguish the signals of SGWB and RGW. The RGW signals on high frequency gravitational-wave detector have been discussed [79]. In this paper, we will study the SGWB signals on high frequency gravitational-wave detector.

The paper is organized as follows. In Sect. 2, we will investigate energy density spectrum and amplitude of SGWB in high frequency band $10^{8}-10^{10} \mathrm{~Hz}$. In Sect. 3, we will investigate the expected electromagnetic signals generated by SGWB in the high frequency GW detector. Conclusions and remarks are presented in Sect. 4.

\section{Stochastic gravitational-wave background in high frequency band}

PBH is considered as a fraction of the dark matter. The abundance of the PBH in dark matter is defined as $f=$ $\Omega_{\mathrm{PBH}} / \Omega_{\mathrm{DM}}$. In Ref. [33], the upper limits of the abundance of the PBHs with mass $10^{-5} M_{\odot}, 10^{-6} M_{\odot}$, and $10^{-7} M_{\odot}$ are given as $10^{-1}, 10^{-0.95}$, and $10^{-0.6}$, respectively. As is given in Refs. [67,68], the corresponding GW frequency ranges from $10^{8}$ to $10^{10} \mathrm{~Hz}$ for merging black hole binaries of chirp mass in $10^{-5} M_{\odot}-10^{-7} M_{\odot}$. To investigate the signals of SGWB generated by merger of the PBH binary, we should first obtain the upper limit of amplitude of the SGWB.

The tidal force from a third PBH will cause the PBH binary loss energy and merge finally. The probability of mergers of PBHs in the cosmic time interval $(t, t+\mathrm{d} t)$ is given as [80]

$\mathrm{d} P_{t}=\frac{3}{58}\left(-\left(\frac{t}{T}\right)^{\frac{3}{8}}+\left(\frac{t}{T}\right)^{\frac{3}{37}}\right) \frac{\mathrm{d} t}{t}, t<t_{c}$,

$\mathrm{d} P_{t}=\frac{3}{58}\left(\frac{t}{T}\right)^{\frac{3}{8}}\left(-1+\left(\frac{t}{t_{c}}\right)^{-\frac{29}{56}} f^{-\frac{29}{8}}\right) \frac{\mathrm{d} t}{t}, t \geq t_{c}$,

where $T=\frac{3}{170} \frac{c^{5} \bar{x}^{4}}{\left(G M_{\mathrm{PBH}}\right)^{3} f^{4}}, t_{c}=\frac{3}{170} \frac{c^{5} \bar{x}^{4} f^{25 / 3}}{\left(G M_{\mathrm{PBH}}\right)^{3}}, c$ is the speed of light, $G$ is gravitational constant, $M_{\mathrm{PBH}}$ is the mass of the PBH, $f$ is the abundance of PBHs in dark mater. $\bar{x}=\left(\frac{M_{\mathrm{PBH}}}{\rho_{\mathrm{PBH}}\left(z_{\text {eq }}\right)}\right)^{\frac{1}{3}}$ is the physical mean separation of PBHs at matter-radiation equality with the redshift $z=z_{\mathrm{eq}}$. Then the event rate of mergers of PBHs is given as

$R_{\mathrm{PBH}}(z)=\frac{3 H_{0}^{2}}{8 \pi G} \frac{f \Omega_{\mathrm{DM}}}{M_{\mathrm{PBH}}} \frac{\mathrm{d} P_{t}}{\mathrm{~d} t}$,

where $H_{0}=67.8 \mathrm{~km} \mathrm{~s}^{-1} \mathrm{Mpc}^{-1}$ is the Hubble constant, $\Omega_{\mathrm{DM}}=0.270$ is the fraction of dark matter. The redshift $z$ is relevant to the cosmic time $t$, i.e., $t=t_{0}-\frac{1}{H_{0}} \int_{0}^{z} \frac{\mathrm{d} z^{\prime}}{\left(1+z^{\prime}\right) E\left(z^{\prime}\right)}$, where $t_{0}$ is the age of the universe, $E(z) \equiv H(z) / H_{0}=$ $\sqrt{\Omega_{M}(1+z)^{3}+\Omega_{\Lambda}}, \Omega_{M}=0.307$ is the fraction of matter [81].

The energy density spectrum of SGWB produced by the merger of PBH binaries [82] can be calculated by using the merger rate of PBHs (3),

$\Omega_{\mathrm{GW}}(v)=\frac{v}{\rho_{c} H_{0}} \int_{0}^{z_{\text {sup }}} \frac{R_{\mathrm{PBH}}(z)}{(1+z) E(z)} \frac{\mathrm{d} E_{\mathrm{GW}}}{\mathrm{d} v_{s}}\left(v_{s}\right) \mathrm{d} z$,

where $\rho_{c}=3 H_{0}^{2} c^{2} / 8 \pi G$ denotes the present value of the critical energy density of the universe, $\mathrm{d} E_{\mathrm{GW}} / \mathrm{d} v_{s}\left(v_{s}\right)$ denotes the energy spectrum of BBH with non-precessing spin correction $[67,68], z_{\text {sup }}=\min \left(z_{\max }, v_{\text {cut }} / v-1\right)\left(z_{\max }\right.$ denotes the maximum redshift given by the PBH model and $v_{\text {cut }}$ is the cut-off frequency given by the energy spectrum of 


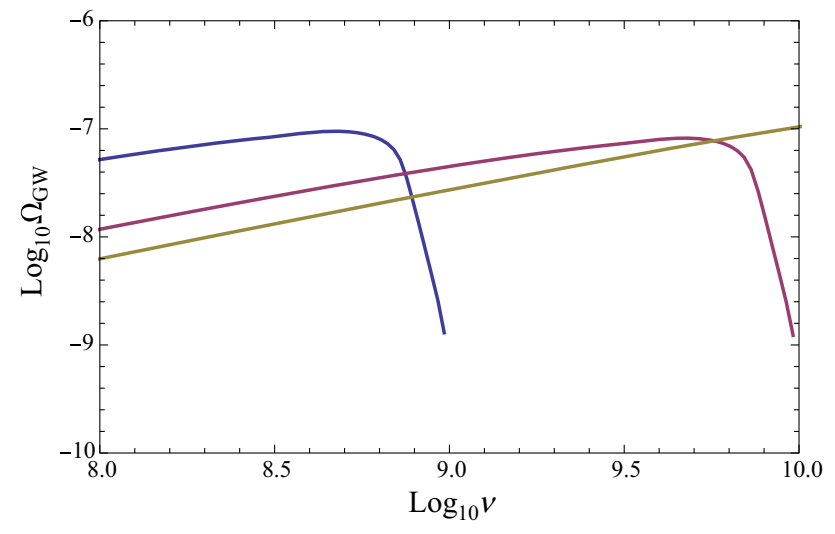

Fig. 1 The SGWB energy density spectrum for various chirp masses, $M_{c}=10^{-5} M_{\odot}, 10^{-6} M_{\odot}, 10^{-7} M_{\odot}$, shown by the blue curve, the purple curve, and the brown curve, respectively

$\mathrm{BBH})$. Here, $v$ is the frequency of the observer frame, $v_{\mathrm{s}}$ is the frequency of the source frame and satisfies the relation $v_{\mathrm{S}}=(1+z) v, R_{\mathrm{PBH}}(z) / 1+z$ denotes that the event rate of $\mathrm{PBH}$ mergers in the source frame is converted to the observer frame.

In this paper, for simplicity, we consider the case that the mass components of the PBH binary are equal to each other. Then one can obtain the energy density spectrum of SGWB by substituting Eqs. (1), (2), (3) into Eq. (4). One should notice the upper limit of integration (4); $z_{\text {sup }}$ takes the value of $v_{\text {cut }} / v-1$ since the PBHs are formed in the early stage of the universe, i.e. $z_{\max } \gg v_{\text {cut }} / v-1$. Also considering the upper limit of the abundance of the PBH given in Ref. [33], we obtain the energy density spectrum of SGWBs generated by the merger of a PBH binary with chirp mass $10^{-5} M_{\odot}$, $10^{-6} M_{\odot}$, and $10^{-7} M_{\odot}$. It is shown in Fig. 1. In the frequency band $10^{8}-10^{10} \mathrm{~Hz}$, the $\Omega_{\mathrm{GW}}$ is relevant to the frequency and the abundance of PBH in dark matter, and the upper limit of $\Omega_{\mathrm{GW}}$ is around $10^{-7}$.

The amplitude of the SGWB generated by the merger of PBH binaries should depend on the angle between the merging plane and the propagating direction of SGWB $[67,68]$. However, since the GW detectors located on earth could not precisely determine the direction of the GWs and the distance between the source of the SGWB and GW detector is high enough, we could neglect such an effect and assume approximately that the SGWB is a plane wave while it arrives at the GW detector. The energy density spectrum of GW can be defined by $\Omega_{\mathrm{GW}}=\left\langle\rho_{\mathrm{GW}}\right\rangle / \rho_{c}$, where $\left\langle\rho_{\mathrm{GW}}\right\rangle=\frac{1}{32 \pi G}\left\langle\dot{h}_{a b} \dot{h}^{a b}\right\rangle$; the angular bracket denotes a spatial average over a few wavelengths. Then, by making use of the plane wave approximation, we can derive the amplitude of the SGWB from its energy density spectrum

$h(v)=\sqrt{\frac{3 H_{0}^{2} \Omega_{\mathrm{GW}}(v)}{\pi^{2} v^{2}}}$.

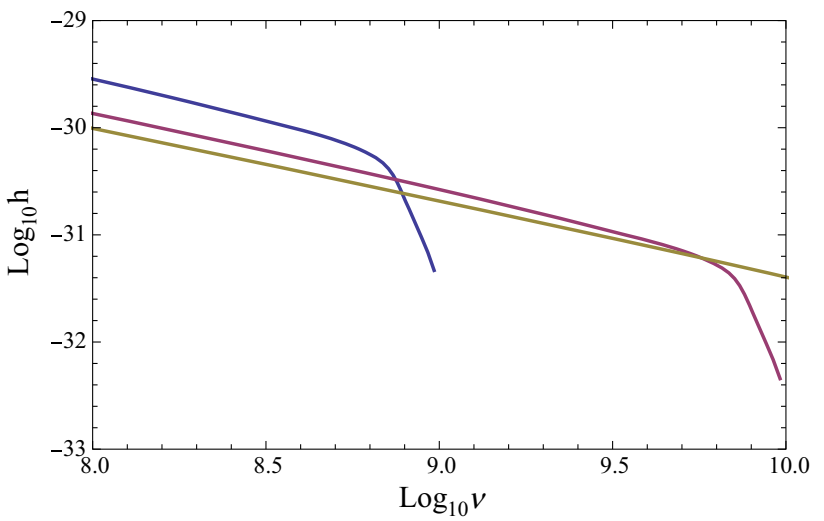

Fig. 2 The amplitude of SGWB for various chirp mass $M_{c}=$ $10^{-5} M_{\odot}, 10^{-6} M_{\odot}, 10^{-7} M_{\odot}$, shown by the blue curve, the purple curve, and the brown curve, respectively

In the high frequency band $10^{8}-10^{10} \mathrm{~Hz}$, the upper limit of the amplitude of the SGWB is given in Fig. 2. One can find from Fig. 2 that the upper limit of the amplitude of SGWB ranges from $10^{-31.5}$ to $10^{-29.5}$ in the frequency band $10^{8}-10^{10} \mathrm{~Hz}$. The optimal response frequency of the high frequency GW (HFGW) detector suggested by $\mathrm{Li}$ and Baker $[69,73-76]$ is around $5 \times 10^{9} \mathrm{~Hz}$. The amplitude of the SGWB could reach up to $10^{-31}$ around the frequency $5 \times 10^{9} \mathrm{~Hz}$, which enters the detectable region of Li-Baker's HFGW detector. For comparison, in the canonical single-field slow-roll inflation models, the amplitude of RGWs obtained in Refs. [77-79] ranges from $10^{-34}$ to $10^{-36}$ in the frequency band $10^{8}-10^{10} \mathrm{~Hz}$. Especially, the amplitude of RGW is around $10^{-36}$ with frequency $5 \times 10^{9} \mathrm{~Hz}$. Therefore, the SGWB generated by a merger of PBH binaries is more likely to be detected by Li-Baker's HFGW detector.

\section{Expected response of electromagnetic resonant in the HFGW detector}

The HFGW detector detects perturbative signal photon flux that is generated by resonance effect between GW and background electromagnetic field. The suggested experimental device is composed of the electromagnetic (EM) field [i.e., Gaussian Beam (GB)] and static magnetic field [83]. The experimental parameters are as follows: $\bar{B}_{y}^{0}=10 \mathrm{~T}$ denotes the background static magnetic field pointing along the $y$ axis localized in a region that is $-l_{1} \leq z \leq l_{2}$ along the $z$-zxis, where $l_{1}=5.7 \mathrm{~m}$ and $l_{2}=0.3 \mathrm{~m}$. The systematic parameters of the Li-Baker detector can be achieved by the present scientific technology [69,73-76]. The general form of GBs propagating along the $z$-axis in a fundamental frequency mode could be written as 


$$
\begin{aligned}
\psi= & \frac{\psi_{0}}{\sqrt{1+\left(\frac{z}{z_{0}}\right)^{2}}} \exp \left(-\frac{r^{2}}{W^{2}}\right) \\
& \times \exp \left\{i\left(\kappa_{e} z-\omega_{e} t\right)-\tan ^{-1} \frac{z}{z_{0}}+\frac{\kappa_{e} r^{2}}{2 R}+\delta\right\},
\end{aligned}
$$

where $r^{2}=x^{2}+y^{2}, \kappa_{e}=2 \pi / \lambda_{e}, \omega_{e}=\kappa_{e} c . \lambda_{e}, \kappa_{e}, \omega_{e}$ is the wavelength, wave vector and angle frequency of the EM field of GB, respectively. $\psi_{0} \approx 1.26 \times 10^{3} \mathrm{~V} \mathrm{~m}^{-1}$ denotes the amplitude of the electric field component of GB. $z_{0}=\frac{\pi W_{0}^{2}}{\lambda_{e}}$ is the Rayleigh size of the GB, where $W_{0}=0.05 \mathrm{~m}$ denotes the waist of GB, i.e., the minimum spot radius along the $z$ axis. We have $W=W_{0} \sqrt{1+\left(\frac{z}{z_{0}}\right)^{2}}, R=z+\frac{z_{0}^{2}}{z}$ is the curvature radius of the wave fronts of the GB at $z$-axis and the $z$-axis is the symmetrical axis of the GB. $\delta=\frac{\pi}{2}$ is the phase difference between GB and the resonant components of HFGW.

For simplicity, we set the background electric field $E_{x}^{0}=$ $\psi=\psi_{x}$ and $E_{z}^{0}=0$. Then, from Maxwell's equations, we can obtain the components of the background EM field,

$E_{y}^{0}=2 x\left(\frac{1}{W^{2}}-\frac{i \kappa_{e}}{2 R}\right) \int E_{x}^{0} \mathrm{~d} y$,

$B_{z}^{0}=\frac{i}{\omega_{e}}\left(\frac{\partial E_{x}^{0}}{\partial y}-\frac{\partial E_{y}^{0}}{\partial x}\right)$.

The GW will cause a fluctuation of the spacetime. When it arrives into the HFGW detector, the background electromagnetic field will change according to the Maxwell equations in curved spacetime. From the difference between the Maxwell equations in curved spacetime and in Minkowski spacetime one will deduce the first-order perburbative EM field on transverse axis. When the angle frequency of the background EM field $\omega_{e}$ is equal to the angle frequency of HFGW $\omega_{g}$, the coherence effect will generate a transverse perturbative photon flux (PPF). According to Refs. [69,73-76], the PPF propagating along $x$-axis is larger than the one that propagates along other directions. Therefore, in this paper we only focus on a photon signal along the $x$-axis for simplicity. The background photon flux (BPF) density along the $x$-axis is given as

$n_{x}^{0}=\frac{1}{\hbar \omega_{e}}\left\langle\frac{1}{\mu_{0}}\left(E_{y}^{0} B_{z}^{0}\right)\right\rangle$,

where $\mu_{0}$ is the vacuum permeability, $\hbar \omega_{e}$ denotes the energy of a single photon, and the angular bracket denotes the average over time.

The first-order perturbed electric field $E_{y}^{(1)}$ can be derived from the Maxwell equations. It is given as [69]

$$
\begin{aligned}
E_{y}^{(1)}= & -\frac{1}{2} h\left(v_{g}\right) \bar{B}_{y}^{0} \kappa_{g} c\left(z+l_{1}\right) \exp \left[i\left(\kappa_{g} z-\omega_{g} t\right)\right] \\
& +\frac{i}{4} h\left(v_{g}\right) \bar{B}_{y}^{0} c \exp \left[i\left(\kappa_{g} z+\omega_{g} t\right)\right],
\end{aligned}
$$

where $h\left(v_{g}\right)$ is amplitude of HFGW, $\kappa_{g}$ is the wave vector of HFGW. By making use of Eqs. (8) and (10), the transverse first-order PPF density along the $x$-axis is given as

$n_{x}^{1}=\frac{1}{\hbar \omega_{e}}\left\langle\frac{1}{\mu_{0}}\left(E_{y}^{1} B_{z}^{0}\right)\right\rangle$.

Then the BPF and PPF along the $x$-axis can be derived from Eqs. (9) and (11),

$N_{x}^{0}=\iint_{\Delta s} n_{x}^{0} \mathrm{~d} y \mathrm{~d} z$

$N_{x}^{1}=\iint_{\Delta s} n_{x}^{1} \mathrm{~d} y \mathrm{~d} z$

where $\Delta s$ is a "typical receiving surface" on the yoz plane, and $0<y<0.1 \mathrm{~m}, 0<z<0.3 \mathrm{~m}$.

Substituting Eqs. (5), (10), and (11) into Eq. (13), and using the upper limit of amplitude of the SGWB, we can obtain the upper limit of the transverse PPF generated by the SGWB with the high frequency band, $10^{8}-10^{10} \mathrm{~Hz}$. The transverse PPF $\left.N_{x}^{1}\right|_{x=0.035 \mathrm{~m}}$ is shown in Fig. 3. One can find from Fig. 3 that the upper limit of the transverse PPF $\left.N_{x}^{1}\right|_{x=0.035 \mathrm{~m}}$ ranges from 1 to $10^{2} \mathrm{~s}^{-1}$, approximately. At optimal response frequency $5 \times 10^{9} \mathrm{~Hz}$, the transverse PPF could reach up to $6 \mathrm{~s}^{-1}$ with the energy around $10^{-5} \mathrm{eV}$. For comparison, the transverse PPF generated by RGW [79] and SGWB is shown in Fig. 4. One can find from Fig. 4 that the transverse PPF generated by RGW (lower curve) can vary from $10^{-2}$ to $10^{-4} \mathrm{~s}^{-1}$ in high frequency band $10^{8}-10^{10} \mathrm{~Hz}$. At optimal response frequency $5 \times 10^{9} \mathrm{~Hz}$, the transverse

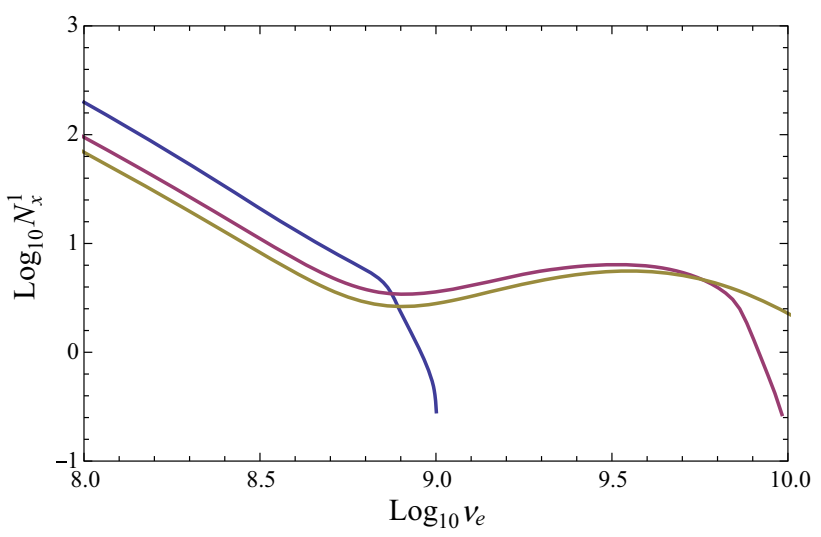

Fig. 3 The transverse PPF $\left.N_{x}^{1}\right|_{x=0.035 \mathrm{~m}}$ generated by the SGWB with high frequency band $10^{8}-10^{10} \mathrm{~Hz}$. The blue curve, the purple curve, and the brown curve correspond to the mergers of black hole binaries with various chirp masses, $M_{c}=10^{-5} M_{\odot}, 10^{-6} M_{\odot}, 10^{-7} M_{\odot}$, respectively 


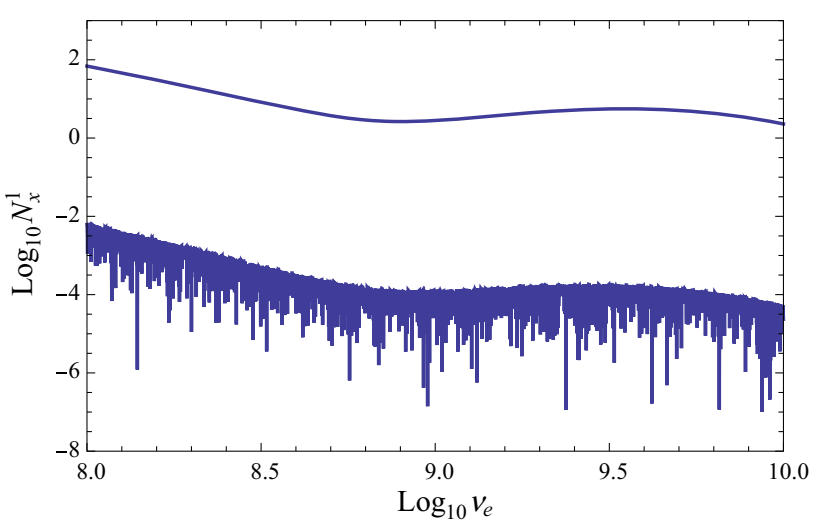

Fig. 4 The transverse PPF $\left.N_{x}^{1}\right|_{x=0.035 \mathrm{~m}}$ generated by RGW and SGWB. The lower curve is the transverse PPF generated by RGW which is predicted by the canonical single-field slow-roll inflation models. The upper curve is the transverse PPF generated by the SGWB correspond to the mergers of black hole binaries with chirp mass $M_{c}=10^{-7} M_{\odot}$

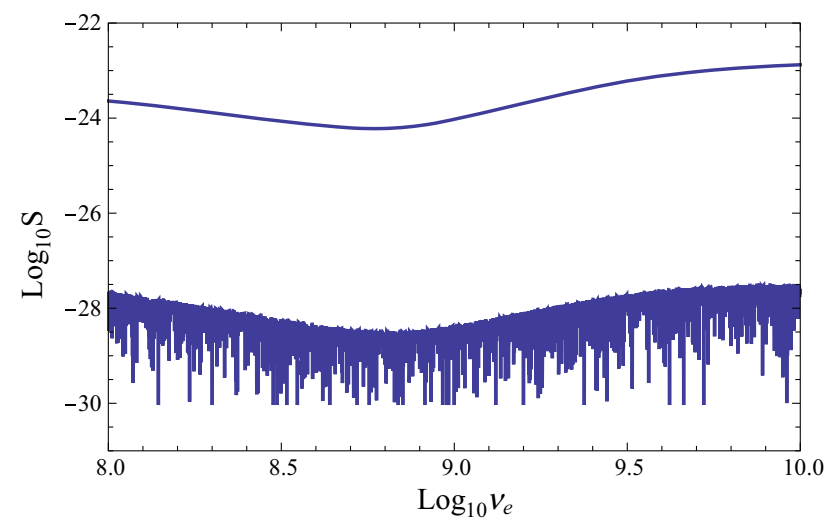

Fig. 5 The ratio of the transverse PPF $N_{x}^{1}$ to the BPF $N_{x}^{0}$ in the high frequency band, i.e., $S=\left.N_{x}^{1}\right|_{x=0.035 \mathrm{~m} /} /\left.N_{x}^{0}\right|_{x=0.035 \mathrm{~m}}$ for RGW and SGWB. The lower curve is the ratio $S$ derived by RGW which is predicted by the canonical single-field slow-roll inflation models. The upper curve is the ratio $S$ derived by the SGWB corresponding to the mergers of black hole binaries with chirp mass $M_{c}=10^{-7} M_{\odot}$

PPF could only reach up to $10^{-4} \mathrm{~s}^{-1}$, while the transverse PPF generated by SGWB could reach up to $6 \mathrm{~s}^{-1}$. Therefore, compared to RGW, the SGWB in the high frequency band $10^{8}-10^{10} \mathrm{~Hz}$ is more likely to be detected by Li-Baker's HFGW detector.

The ratio of the transverse PPF $N_{x}^{1}$ to the BPF $N_{x}^{0}$ in the high frequency band, i.e., $S=N_{x}^{1} /\left.N_{x}^{0}\right|_{x=0.035 \mathrm{~m}}$, describes the basic feature of the Li-Baker HFGW detector. It is shown in Fig. 5. The ratio derived by the SGWB is 4 orders of magnitude higher than the ratio derived by RGW.

\section{Conclusion and remarks}

The black hole with a mass of less than $1 M_{\odot}$ could not have an astrophysical origin, it may be formed at an early stage of the universe. Investigating the SGWB generated by the merger of these black hole binaries could be helpful for us to understand the primordial origin of black holes. In this paper, by applying the current most stringent constraints on the abundance of PBH in dark matter, we have derived the SGWB energy density spectrum generated by the merger of PBH binary with equal mass components. As is shown in Fig. 1, the upper limit of the energy density spectrum of SGWB is around $10^{-7}$. By making use of the plane wave approximation, the upper limit of the amplitude of the SGWB is shown in Fig. 2. It ranges from $10^{-31.5}$ to $10^{-29.5}$. Especially, at the optimal response frequency of HFGW detector, i.e., $5 \times 10^{9} \mathrm{~Hz}$, the amplitude of the SGWB could reach up to $10^{-31}$, which enters the detectable region of Li-Baker's HFGW detector $[69,73-76]$. The upper limit of the PPF $\left.N_{x}^{1}\right|_{x=0.035 \mathrm{~m}}$ generated by the SGWB in the high frequency band $10^{8}-10^{10} \mathrm{~Hz}$ is shown in Fig. 3, which ranges from 1 to $10^{2} \mathrm{~s}^{-1}$ approximately. At the optimal response frequency, $5 \times 10^{9} \mathrm{~Hz}$, the HFGW detector could receive 6 photons in a second with the energy around $10^{-5} \mathrm{eV}$. It is shown in Fig. 4 that the transverse PPF generated by the RGW which is predicted by the canonical single-field slow-roll inflation models is sufficiently lower than the one generated by the SGWB in the high frequency band $10^{8}-10^{10} \mathrm{~Hz}$. The ratio of the transverse PPF to the BPF, i.e., $S=\left.N_{x}^{1}\right|_{x=0.035 \mathrm{~m}} /\left.N_{x}^{0}\right|_{x=0.035 \mathrm{~m}}$, is shown in Fig. 5. Our results indicate that the SGWB in the high frequency band $10^{8}-10^{10} \mathrm{~Hz}$ is more likely to be detected by the Li-Baker HFGW detector.

Acknowledgements We thank Dr. S. Wang, Prof. H. Wen and Prof. F.-Y. Li for helpful discussions. This work has been supported by the National Natural Science Fund of China (Grant No. 11775038 and 11647307) and the Fundamental Research Funds for the Central Universities (Project No. 106112017CDJXFLX0014).

Open Access This article is distributed under the terms of the Creative Commons Attribution 4.0 International License (http://creativecomm ons.org/licenses/by/4.0/), which permits unrestricted use, distribution, and reproduction in any medium, provided you give appropriate credit to the original author(s) and the source, provide a link to the Creative Commons license, and indicate if changes were made.

Funded by $\mathrm{SCOAP}^{3}$.

\section{References}

1. S.B. Giddings, S. Thomas, Phys. Rev. D 65, 056010 (2002)

2. A. Celotti, J.C. Miller, D.W. Sciama, Class. Quantum Gravity 16, A3 (1999)

3. Y.B. Zeldovich, I.D. Novikov, Sov. Astron. 10, 602 (1967)

4. B.J. Carr, S.W. Hawking, Mon. Not. R. Astron. Soc. 168, 399 (1974)

5. B.J. Carr, Astrophys. J. 201, 1 (1975)

6. B.J. Carr, Astrophys. J. 206, 8 (1976)

7. S. Clesse, J. Garca-Bellido, Phys. Rev. D 92, 023524 (2015)

8. A.D. Dolgov, S.I. Blinnikov, Phys. Rev. D 89, 021301 (2014)

9. D. Blais, C. Kiefer, D. Polarski, Phys. Lett. B 535, 11 (2002)

10. M.Y. Khlopov, Res. Astron. Astrophys. 10, 495 (2010) 
11. P.H. Frampton, M. Kawasaki, F. Takahashi, T.T. Yanagida, J. Cosmol. Astropart. Phys. 1004, 023 (2010)

12. S. Blinnikov, A. Dolgov, N. Porayko, K. Postnov, JCAP 11, 036 (2016)

13. A. Tan et al. (PandaX-II), Phys. Rev. Lett. 117, 121303 (2016)

14. D.S. Akerib et al. (LUX), Phys. Rev. Lett. 116, 161301 (2016)

15. J.A. Munoz et al., Astrophys. J. 742, 67 (2011)

16. B.P. Abbott et al. (LIGO Scientific Collaboration and Virgo Collaboration), Phys. Rev. Lett. 116, 061102 (2016)

17. B.P. Abbott et al. (LIGO Scientific Collaboration and Virgo Collaboration), Phys. Rev. Lett. 116, 241103 (2016)

18. B.P. Abbott et al. (LIGO Scientific Collaboration and Virgo Collaboration), Phys. Rev. Lett. 118, 221101 (2017)

19. C.W. Misner, K.S. Thorne, J.A. Wheeler, Gravitation (W. H. Freeman, San Francisco, 1973)

20. N. Chamel, P. Haensel, J.L. Zdunik, A.F. Fantina, Int. J. Mod. Phys. E 22, 1330018 (2013)

21. J. Garcia-Bellido, A. Linde, D. Wands, Phys. Rev. D 54, 6040 (1996)

22. K. Jedamzik, J.C. Niemeyer, Phys. Rev. D 59, 124014 (1999)

23. T. Suyama, T. Tanaka, B. Bassett, H. Kudoh, Phys. Rev. D 71, 063507 (2005)

24. T. Suyama, T. Tanaka, B. Bassett, H. Kudoh, J. Cosmol. Astropart. Phys. 04, 001 (2006)

25. K. Kohri, C.-M. Lin, T. Matsuda, Phys. Rev. D 87, 103527 (2013)

26. M. Kawasaki, N. Kitajima, T.T. Yanagida, Phys. Rev. D 87, 063519 (2013)

27. E.V. Bugaev, P.A. Klimai, Int. J. Mod. Phys. D 22, 1350034 (2013)

28. A. Dolgov, J. Silk, Phys. Rev. D 47, 4244 (1993)

29. A.D. Dolgov, M. Kawasaki, N. Kevlishvili, Nucl. Phys. B 807, 229 (2009)

30. MYu. Khlopov, R.V. Konoplich, S.G. Rubin, A.S. Sakharov, Grav. Cosmol. 2, S1 (1999)

31. S.G. Rubin, MYu. Khlopov, A.S. Sakharov, Grav. Cosmol. 6, 51 (2000)

32. V.I. Dokuchaev, Y.N. Eroshenko, S.G. Rubin, arXiv:0709.0070 [astro-ph]

33. B. Carr, F. Kuhnel, M. Sandstad, Phys. Rev. D 94, 083504 (2016)

34. S.W. Hawking, Nature 248, 30 (1974)

35. B.P. Abbott et al. (Virgo, LIGO Scientific), Astrophys. J. 818, L22 (2016)

36. K. Belczynski, D.E. Holz, T. Bulik, R. O’Shaughnessy, Nature 534, 512 (2016)

37. K. Belczynski, M. Dominik, T. Bulik, R. O'Shaughnessy, C. Fryer, D.E. Holz, Astrophys. J. 715, L138 (2010)

38. S. Bird, I. Cholis, J.B. Muoz, Y. Ali-Hamoud, M. Kamionkowski, E.D. Kovetz, A. Raccanelli, A.G. Riess, Phys. Rev. Lett. 116, $201301(2016)$

39. S. Clesse, J. Garca-Bellido, Phys. Dark Univ. 15, 002 (2017)

40. M. Sasaki, T. Suyama, T. Tanaka, S. Yokoyama, Phys. Rev. Lett. 117, 061101 (2016)

41. A. Kashlinsky, Astrophys. J. 823, L25 (2016)

42. N. Bartolo et al., JCAP 12, 026 (2016)

43. L. Chen, Q.-G. Huang, K. Wang, JCAP 12, 044 (2016)

44. S. Wang, Y.-F. Wang, Q.-G. Huang, T.G.F. Li, arXiv:1610.08725

45. V. Ferrari, S. Matarrese, R. Schneider, Mon. Not. R. Astron. Soc. 303, 247 (1999)
46. T. Regimbau, J.A. de Freitas Pacheco, Astron. Astrophys. 376, 381 (2001)

47. R. Schneider et al., Mon. Not. R. Astron. Soc. 324, 797 (2001)

48. A.J. Farmer, E.S. Phinney, Mon. Not. R. Astron. Soc. 346, 1197 (2003)

49. T. Regimbau, J.A. de Freitas Pacheco, Astrophys. J. 642, 455 (2006)

50. T. Regimbau, V. Mandic, Class. Quantum Gravity 25, 184018 (2008)

51. T. Regimbau, S.A. Hughes, Phys. Rev. D 79, 062002 (2009)

52. T. Regimbau, Res. Astron. Astrophys. 11, 369 (2011)

53. X.J. Zhu, E. Howell, T. Regimbau, D. Blair, Z.H. Zhu, Astrophys. J. 739, 86 (2011)

54. C. Wu, V. Mandic, T. Regimbau, Phys. Rev. D 85, 104024 (2012)

55. S. Marassi, R. Schneider, G. Corvino, V. Ferrari, S. Portegies Zwart, Phys. Rev. D 84, 124037 (2011)

56. P.A. Rosado, Phys. Rev. D 84, 084004 (2011)

57. C.J. Wu, V. Mandic, T. Regimbau, Phys. Rev. D 87, 042002 (2013)

58. A. Sesana et al., Astrophys. J. 623, 23 (2005)

59. A. Sesana, A. Vecchio, C.N. Colacino, Mon. Not. R. Astron. Soc. 390, 192 (2008)

60. T. Regimbau, J.A. de Freitas Pacheco, Astron. Astrophys. 447, 1 (2006)

61. B.P. Abbott et al. (Virgo, LIGO Scientific), Phys. Rev. Lett. 116, 131102 (2016)

62. G. Hobbs, Class. Quantum Gravity 25, 114032 (2008)

63. R. Nan et al., Int. J. Mod. Phys. D 20, 989 (2011)

64. R.M. Shannon et al., Science 349, 1522 (2015)

65. L. Lentati et al. (EPTA Collaboration), Mon. Not. Astron. Soc. 453, $2576(2015)$

66. P. Amaro-Seoane et al., Class. Quantum Gravity 29, 124016 (2012)

67. P. Ajith et al., Phys. Rev. D 77, 104017 (2008)

68. P. Ajith et al., Phys. Rev. Lett. 106, 241101 (2011)

69. F.Y. Li, R.M.L. Baker Jr., Z.-Y. Fang, G.V. Stephenson, Z.Y. Chen, Eur. Phys. J. C 56, 407 (2008)

70. M.E. Gertsenshtein, Sov. Phys. JETP 14, 84 (1962)

71. G. Raffelt, L. Stodolsky, Phys. Rev. D 37, 1237 (1988)

72. A.D. Dolgov, D. Ejlli, JCAP 12, 003 (2012)

73. F.-Y. Li, N. Yang, Z.-Y. Fang, R.M.L. Baker Jr., G.V. Stephenson, H. Wen, Phys. Rev. D 80, 064013 (2009)

74. J. Li, K. Lin, F.-Y. Li, Y.-H. Zhong, Gen. Relativ. Gravit. 43, 2209 (2011)

75. H. Wen, F.-Y. Li, Z.-Y. Fang, Phys. Rev. D 89, 104025 (2014)

76. H. Wen, F.-Y. Li, Z.-Y. Fang, A. Beckwith, Eur. Phys. J. C 74, 2998 (2014)

77. H.X. Miao, Y. Zhang, Phys. Rev. D 75, 104009 (2007)

78. X.J. Liu, W. Zhao, Y. Zhang, Z.H. Zhu, Phys. Rev. D 93, 024031 (2016)

79. X. Li, S. Wang, H. Wen, Chin. Phys. C 40, 085101 (2016)

80. M. Sasaki, T. Suyama, T. Tanaka, S. Yokoyama, Phys. Rev. Lett. 117, 061101 (2016)

81. Planck Collaboration, Astron. Astrophys. 594, A13 (2016)

82. B. Allen, J.D. Romano, Phys. Rev. D 59, 102001 (1999)

83. J. Li, L. Zhang, K. Lin, H. Wen, Int. J. Theor. Phys. 55, 3506 (2016) 